\title{
EMG activity during whole body vibration: motion artifacts or stretch reflexes?
}

\author{
Ramona Ritzmann - Andreas Kramer · \\ Markus Gruber · Albert Gollhofer • \\ Wolfgang Taube
}

\begin{abstract}
The validity of electromyographic (EMG) data recorded during whole body vibration (WBV) is controversial. Some authors ascribed a major part of the EMG signal to vibration-induced motion artifacts while others have interpreted the EMG signals as muscular activity caused at least partly by stretch reflexes. The aim of this study was to explore the origin of the EMG signal during WBV using several independent approaches. In ten participants, the latencies and spectrograms of stretch reflex. responses evoked by passive dorsiflexions in an ankle ergometer were compared to those of the EMG activity of four leg muscles during WBV. Pressure application to the muscles was used to selectively reduce the stretch reflex, thus permitting to distinguish stretch reflexes from other signals. To monitor motion artifacts, dummy electrodes were placed close to the normal electrodes. Strong evidence for stretch reflexes was found: the latencies of the stretch reflex responses evoked by dorsiflexions were almost identical to the supposed stretch reflex responses during vibration (differences of less than $1 \mathrm{~ms}$ ). Pressure application significantly reduced the amplitude of both the supposed stretch reflexes during vibration (by $61 \pm 17 \%$, $p<0.001)$ and the stretch reflexes in the ankle ergometer
\end{abstract}

Communicated by Arnold de Haan.

R. Ritzmann $(\bigotimes)$ - A. Kramer · A. Gollhofer - W. Taube Department of Sport and Sport Science, University of Freiburg, Schwarzwaldstraße 175, 79117 Freiburg, Germany e-mail: ramona.ritzmann@sport.uni-freiburg.de

M. Gruber

Department of Training and Movement Science, University of Potsdam, Potsdam, Germany (by $56 \pm 13 \%, p<0.01$ ). The dummy electrodes showed almost no activity during WBV $(7 \pm 4 \%$ of the corresponding muscle's iEMG signal). The frequency analyses revealed no evidence of motion artifacts. The present results support the hypothesis of WBV-induced stretch reflexes. Contribution of motion artifacts to the overall EMG activity seems to be insignificant.

Keywords Electromyography - Afferent .

Pressure application - Frequency analysis - Spectrogram . Latency

\section{Introduction}

Whole body vibration (WBV) platforms have become popular training devices in recreational and athletic training as well as for rehabilitation purposes. There are various studies investigating the acute (Abercromby et al. 2007; Cochrane and Stannard 2005; de Ruiter et al. 2003b; Rittweger et al. 2000, 2003; Torvinen et al. 2002) and longterm (Blottner et al. 2006; de Ruiter et al. 2003a; Kvorning et al. 2006) effects of WBV training. Some of these studies (Abercromby et al. 2007; Torvinen et al. 2002) used surface electromyography (EMG) to measure the level of the neuromuscular activity during WBV in order to evaluate training parameters such as vibration type, vibration frequency, vibration amplitude or body position. However, the nature and validity of EMG recordings during WBV has to be examined carefully before any deductions can be drawn based on the EMG data. In case of WBV-induced stretch reflex responses, adaptations following WBV exercises can be interpreted on a neuromuscular basis. However, a potential source of error is the occurrence of motion artifacts caused by the applied vibration. To our 
knowledge, there are only two studies addressing the problem of motion artifacts. Abercromby et al. (2007) studied the acute effects of vibration type (rotational vs. vertical), contraction type (eccentric vs. concentric vs. isometric) and knee angle during WBV on the electromyographic activity of four leg muscles. The sharp peaks at the vibration frequency and its harmonics in the spectrograms of the recorded EMG were interpreted as an evidence for motion artifacts. Based on this finding, the authors concluded that band-stop filters should be applied to EMG signals recorded during WBV to eliminate those frequency peaks. Fratini et al. (2009) studied the relevance of motion artifacts during WBV by placing electrodes on the patella, assuming that any signal recorded with those electrodes had to be an artifact. In the spectrograms, they observed sharp peaks at the vibration frequency and its harmonics too, even in the spectrogram derived from the patella electrodes. This finding led them to the same conclusion as Abercromby et al. (2007). However, due to methodological constraints, neither of these studies can provide conclusive evidence for the assumption that a considerable amount of the EMG signal is due to motion artifacts. Abercromby et al. (2007) only looked at the spectrogram of the EMG signal and drew conclusions that a spectrogram cannot provide on mathematical grounds, i.e. they neglected the fact that the observed shape need not necessarily be caused by artifacts. Fratini et al. (2009) overlooked the fact that electrodes placed on the patella do not exclusively monitor artifacts but also crosstalk from surrounding muscles.

Various studies have suggested that WBV evokes muscle contractions via stretch reflexes (Kvorning et al. 2006; Mester et al. 2002; Rittweger et al. 2003). The first to demonstrate that vibration can elicit reflex excitation of the soleus muscle of the decerebrate cat was Matthews (1966). In order to investigate the relation between the frequency of vibration and the size of the resulting reflex contraction, he applied vibration to the Achilles tendon (with an amplitude of $10 \mu \mathrm{m}$ ) and concluded that "the primary afferent ending of the muscle spindle is considered to be the receptor whose excitation leads to the reflex response to vibration. The vibration reflex thus appears to be the wellknown stretch reflex, elicited by a rather unusual form of stretching" (Matthews 1966, p. 451). But are these observations applicable to man? The basic conditions for stretch reflexes during WBV are met, namely the stretch velocity (more than $300 \%$ at $30 \mathrm{~Hz}$ ) and the stretch amplitude (1-5 mm, depending on the vibration platform). Gollhofer and Rapp (1993) reported that the magnitude of the stretch reflex was positively correlated to the stretch velocity, and a stretch velocity of $300 \% \mathrm{~s}$ (as used in this study) is well beyond the $100 \%$ used by Gollhofer and Rapp to evoke stretch refiexes. Previous studies have also shown that the proprioceptive system is not only capable to respond very quickly to changes in muscle length but can also repeat their stretch-induced responses with high $(>50 \mathrm{~Hz})$ frequencies (Burke et al. 1976; Roll et al. 1989). Based on these findings, it seems reasonable to assume that WBV may influence muscular activity by eliciting reflex responses caused by repeated stretches of the tendomuscular system. Therefore, in the present study, we hypothesized that the periodic spikes in the EMG signal during WBV are not motion artifacts, but rather stretch reflex responses evoked by the vibration-induced joint rotation. To our knowledge, at the time this study was conducted it was the first one attempting to gather evidence for WBVinduced stretch reflexes.

\section{Methods}

No method is known that can demonstrate on its own whether an EMG signal consists primarily of vibrationinduced motion artifacts or of stretch reflexes. Therefore, it is necessary to use several independent approaches. In combination, the results of the different methods can clarify the severity of motion artifacts and can help to decide whether the characteristics of the EMG signal match those of stretch reflex responses. First, to test whether the movement of the cables and electrodes during WBV caused motion artifacts in the EMG signal, special dummy electrodes were developed, in order to provide a signal that would only be influenced by motion artifacts, without interference from muscle signals. The other three approaches had the same goal, but used a physiological approach and in addition they were designed to verify whether the characteristics of the EMG signal were consistent with the characteristics of a stretch reflex response. For that purpose, frequency spectra and latencies of mechanically evoked stretch reflexes and of the EMG signal during WBV were evaluated and compared. Moreover, pressure application to the soleus muscle via a blood pressure cuff served to selectively reduce the amplitude of the short-latency component of the stretch reflex, allowing an estimation of the stretch reflex contribution to the EMG signal.

\section{Experimental design}

A single-group repeated-measures study design was used, with several protocols examining the nature of the electromyographic activity of four leg muscles and possible vibration-induced motion artifacts. The order of the protocols and test conditions was balanced between participants to control confounding effects such as fatigue. 
Subjects

Ten healthy subjects ( 5 females, 5 males, age $26 \pm 3$ years) volunteered to participate in this study. All participants gave written informed consent to the experimental procedure, which was approved by the ethics committee of the University of Freiburg and in accordance with the latest revision of the Declaration of Helsinki. The participants were healthy with no previous neurological irregularities or injuries of the lower extremity. Their mean ( \pm standard deviation) height and weight were $172 \pm 9 \mathrm{~cm}$ and $65 \pm 13 \mathrm{~kg}$, respectively.

\section{EMG recording}

Bipolar $\mathrm{Ag} / \mathrm{AgCl}$ surface electrodes (Ambu Blue Sensor $\mathrm{P}$, Ballerup, Denmark; diameter $9 \mathrm{~mm}$, center-to-center distance $34 \mathrm{~mm}$ ) were placed over $M$. soleus (SOL), $M$. gastrocnemius medialis (GM), M. tibialis anterior (TA) and M. rectus femoris ( $R F$ ) of the right leg. The longitudinal axes of the electrodes were in line with the presumed direction of the underlying muscle fibers. The reference electrode was placed on the patella. Interelectrode resistance was kept below $2.2 \mathrm{k} \Omega$ by means of shaving, light abrasion and degreasing of the skin with a disinfectant. The EMG signals were transmitted via shielded cables to the amplifier (band-pass filter $3 \mathrm{~Hz}-1 \mathrm{kHz}, 1,000 \times$ amplified; the lower limit of $3 \mathrm{~Hz}$ was chosen in order not to filter the lowest vibration frequency of $5 \mathrm{~Hz}$ ) and recorded with $3,650 \mathrm{~Hz}$ (synchronously and with the same frequency as all other recorded signals). The cables were carefully taped to the skin. The signed integrals of the EMG signals were calculated and set to zero (per trial and muscle) to correct possible offsets. Afterwards, mean and standard deviation (SD) were calculated from signal pieces with a length of one vibration period (from one low point of the vibration platform to the next one) using the semiconductor accelerometer mounted on the vibration platform (fixed with strong glue) as a trigger signal. The iEMG was then calculated as the area of the mean EMG signal with a length of one vibration period, i.e. between 33 and $200 \mathrm{~ms}$, depending on the vibration frequency. The recording and signal processing was done using a custom-made Labviewbased program (IMAGO, Pfisoft) and MATLAB ${ }^{\circledR}$ R2007b.

\section{Vibration conditions}

The vibration platform Galileo Sport (Novotec Medical, Pforzheim, Germany) was used. It generates vibration by oscillating along the sagittal axis. Thus, the further away the feet are positioned from the axis of rotation, the larger the vibration amplitude. For this study, a distance of $21 \mathrm{~cm}$ from the axis of rotation was used, resulting in an amplitude of $4 \mathrm{~mm}$ (i.e. a peak-to-peak displacement of $8 \mathrm{~mm}$ ). The vibration frequency of the Galileo platform is adjustable in steps of $0.5 \mathrm{~Hz}$ within a range of $5-30 \mathrm{~Hz}$. To examine the effects of vibration frequency, steps of $5 \mathrm{~Hz}$ were chosen. During testing, the participants did not wear shoes or socks in order to avoid damping effects due to different footwear. The trial was repeated if any large foot movements due to high-frequency vibration were observed. Vibration exposure during a single trial was limited to $10 \mathrm{~s}$ (up to $3 \mathrm{~s}$ to allow the vibration platform to reach its preset amplitude and frequency and $7 \mathrm{~s}$ of actual recording).

\section{Ankle ergometer}

The custom-made ankle ergometer used to evoke stretch reflex responses in SOL and GM had the following specifications: it was driven by two independent motors which controlled the rotation of the right and left foot pedal. Participants were fixed by a strap-binding system, with their feet resting on the rotation platform of the ergometer standing in an upright position (Fig. 1). The rotation axis of the upper ankle joint coincided with the rotation axis of the platform. An induced dorsiflexion movement at the ankle joint with an amplitude of $6^{\circ}$ and a velocity of $150 \% \mathrm{~s}$ evoked a stretch and hence a refiex response in the triceps surae. The pedal position signal was used to determine the onset of the pedal movement for latency calculations (Gollhofer and Rapp (1993)).

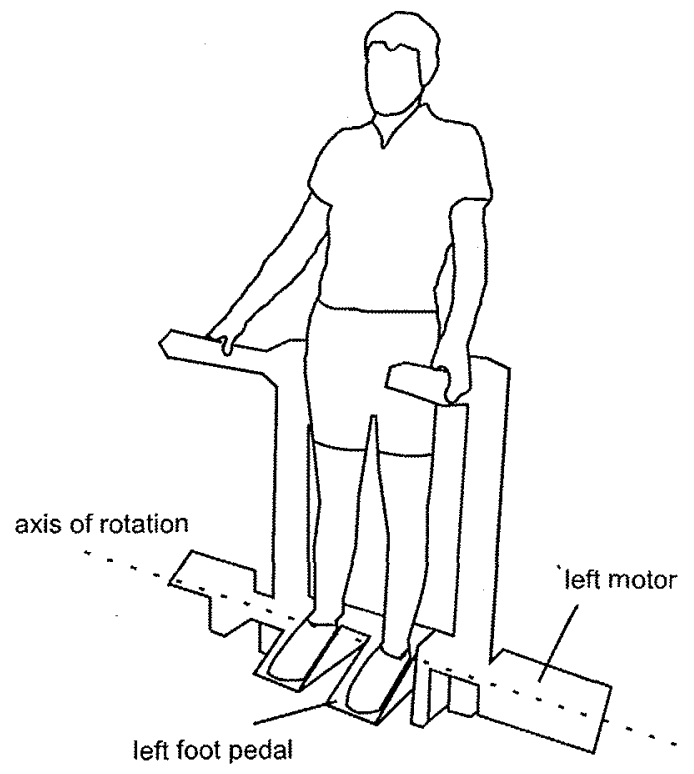

Fig. 1 Illustration of the ankle ergometer used to evoke stretch reflex responses in the triceps surae. The stretch is induced by an ankle dorsiffexion, which in turn is forced by the upwards pedal movement 


\section{Experimental approaches}

The experiment incorporated four different approaches:

1. Dummy electrodes To monitor vibration-induced motion artifacts, three dummy electrodes were positioned close (about $2 \mathrm{~mm}$ away) and parallel to the normal electrodes of $\mathrm{M}$. soleus, $\mathrm{M}$. tibialis anterior and M. rectus femoris. The dummy electrodes consisted of the same bipolar surface electrodes as the ones for normal EMG recording. To insulate them from muscle signals, they were not placed directly on the skin. Instead, the skin was prepared with insulating tape and the dummy electrodes were placed on this tape, resulting in an infinite resistance between the two electrodes. To mimic the skin resistance, the electrodes were connected by a $2.2 \mathrm{k} \Omega$ resistor and their signals were recorded and processed like a normal EMG signal (see EMG recordings). Since it is possible that the body position on the vibration platform and the vibration frequency have an influence on the occurrence of motion artifacts, six frequencies $(5,10,15,20$, $25,30 \mathrm{~Hz})$, three knee angles $\left(5,30,60^{\circ}\right.$ flexion) and two foot positions (forefoot vs. normal stance) were investigated. Altogether, every participant completed 36 WBV trials (6 different frequencies $\times 3$ knee angles $\times 2$ foot positions). To compare the dummy signal to the muscle signal, the ratio of the dummy "iEMG" to the $\mathrm{iEMG}$ of the corresponding muscle (D/M-ratio) was calculated.

2. Frequency analysis In order to compare the frequency spectrum of stretch reflex responses to the frequency spectrum of the EMG signal during WBV, a Fast Fourier Transformation (done with MATLAB ${ }^{\circledR}$ $\mathrm{R} 2007 \mathrm{~b}$ ) was applied to EMG signals with a length of $500 \mathrm{~ms}$ and then averaged for all participants, once for each recorded frequency of the EMG signal during WBV and once for the stretch reflex responses evoked by an ankle ergometer (which were stringed together so that the stretch reflexes were equally distributed during $1 \mathrm{~s}$, resulting in a frequency comparable to the corresponding vibration frequency).

3. Latencies To determine the individual latency of the stretch reflex response, the ankle ergometer was used to evoke stretch reflexes in SOL and GM (with an interstimulus interval of $4 \mathrm{~s}$ ). The stretch reflex latency was defined as the difference between the onset of the dorsiflexion movement (first rise above the neutral level, determined per software) and the onset of the first rise in EMG activity (determined visually). The latency during WBV was defined in a similar manner, namely as the time period from the low point of the vibration platform (coinciding with the beginning of the ankle dorsiflexion and therefore presumably the muscle stretch) to the onset of the first spike in the EMG signal, the supposed stretch reflex. The low point of the vibration platform was determined using the accelerometer fixed on the platform. Whenever the dummy signal did not exclusively consist of background noise but had singular peaks, their "latencies" were determined too. To compare a participant's latencies in the ankle ergometer (representing the latency of the ankle stretch reflex) to the latencies during WBV, the mean and standard deviation of 20 trials in the ankle ergometer and the mean and standard deviation of all vibration trials (36 trials) were calculated. Afterwards, the difference between the stretch reflex latency in the ankle ergometer and the latency of the supposed stretch reflex during WBV was calculated for every participant in SOL and in GM.

4. Cuff experiment Recently, it was demonstrated that pressure induced by a conventional blood pressure cuff instantly reduced the short-latency response of the stretch reflex but not subsequent reflex components (Leukel et al. 2009). In the present study, a conventional blood pressure cuff (Bosch \& Sohn, Jungingen, Germany) was used to mechanically compress the belly of the SOL muscle. The cuff was placed in the middle of the lower leg just above the SOL electrodes. First, the influence of the cuff onto the stretch reflex was tested with the participants sitting in the ankle ergometer. For this purpose, ten stretch reflexes with deflated cuff were compared to ten stretch reflexes with the cuff inflated to $220-240 \mathrm{mmHg}$ (i.e. during $40 \mathrm{~s}$ of pressure application). The same experiment was conducted with the participant sitting on a chair with the feet on the vibration platform (amplitude $2 \mathrm{~mm}$, frequency $22 \mathrm{~Hz}$, duration $10 \mathrm{~s}$ ). The data of the cuff experiment in the ankle ergometer and during WBV were triggered as described above, averaged and the peak-to-peak values of the conditions with inflated cuff were calculated and expressed as a percentage of the peak-to-peak values with deflated cuff.

\section{Statistics}

Paired $t$ tests were used to reveal differences between the latencies (mean as well as SD) during vibration and in the ankle ergometer, respectively between the latencies of the muscle signals and the corresponding dummy signals. Differences between trials with deflated and inflated cuff were examined with paired $t$ tests. The level of significance was set to $p \leq 0.05$ (two-tailed). Group data are presented as means $\pm \mathrm{SD}$ unless otherwise stated. All analyses were executed using SPSS 16.0 (SPSS Inc., Chicago, IL, USA). 
Table 1 Averaged D/M ratios (dummy "iEMG" normalized to the corresponding muscle $\mathrm{IEMG}$ ) of SOL, RF and TA without and during WBV

\begin{tabular}{llll}
\hline & Sol & RF & TA \\
\hline Stance, no vibration & $0.14 \pm 0.07$ & $0.27 \pm 0.19$ & $0.36 \pm 0.15$ \\
$5 \mathrm{~Hz}$ & $0.11 \pm 0.07$ & $0.13 \pm 0.04$ & $0.19 \pm 0.12$ \\
$10 \mathrm{~Hz}$ & $0.08 \pm 0.06$ & $0.12 \pm 0.03$ & $0.01 \pm 0.00$ \\
$15 \mathrm{~Hz}$ & $0.05 \pm 0.04$ & $0.07 \pm 0.03$ & $0.12 \pm 0.04$ \\
$20 \mathrm{~Hz}$ & $0.06 \pm 0.03$ & $0.01 \pm 0.00$ & $0.14 \pm 0.07$ \\
$25 \mathrm{~Hz}$ & $0.07 \pm 0.07$ & $0.03 \pm 0.01$ & $0.14 \pm 0.12$ \\
$30 \mathrm{~Hz}$ & $0.04 \pm 0.02$ & $0.03 \pm 0.01$ & $0.09 \pm 0.08$ \\
Mean (vibration only) & $0.07 \pm 0.05$ & $0.07 \pm 0.02$ & $0.11 \pm 0.07$ \\
\hline
\end{tabular}

Values are mean $\pm S D$

\section{Results}

1. Dummy electrodes The analysis of motion artifacts during WBV with the aid of the dummy electrodes resulted in a mean $\mathrm{D} / \mathrm{M}$ ratio of $0.07 \pm 0.05$ for $\mathrm{SOL}$ (i.e. the average activity of the SOL dummy was $7 \%$ of the SOL activity), $0.07 \pm 0.02$ for RF and $0.11 \pm 0.07$ for TA (Table 1). Furthermore, the $D / M$ ratio decreased when vibration frequency increased. In twothirds of all trials, the dummy signals showed a virtually flat line consisting only of background noise (Fig. 2). However, one-third showed small spikes. The "latencies" of these spikes were unsystematic and irregular in the innersubject and intersubject comparison, which is reflected by the high standard deviations for the dummy "latencies" (see latency results).

2. Frequency analysis The frequency spectra derived from the SOL and GM EMG data of a succession of stretch reflexes evoked in the ankle ergometer showed local maxima at the stimulus frequency and its integer multiples. For example, at a frequency of $20 \mathrm{~Hz}$, peaks with decreasing amplitude would appear at 20,40,60, $80, \ldots \mathrm{Hz}$. The spectrogram of the EMG data of the extensor muscles (SOL, GM and RF) during WBV showed a similar pattern: the main component of the spectrogram consisted of the vibration frequency and its integer multiples. The dummy spectrograms showed no consistent patterns, although some had minor peaks. In Fig. 3, the mean of all participants of the frequency spectra of a series of stretch reflexes with an interstimulus interval of $40 \mathrm{~ms}$ (obtained by stringing together stretch reflex responses evoked in the ankle ergometer in order to get a comparable frequency of $25 \mathrm{~Hz}$, Fig. 3a), the mean of all participants of the frequency spectra of a $25 \mathrm{~Hz}$ vibration (unprocessed EMG signal during WBV of SOL and its dummy, Fig. 3b, d) and a spectrogram of a sawtooth
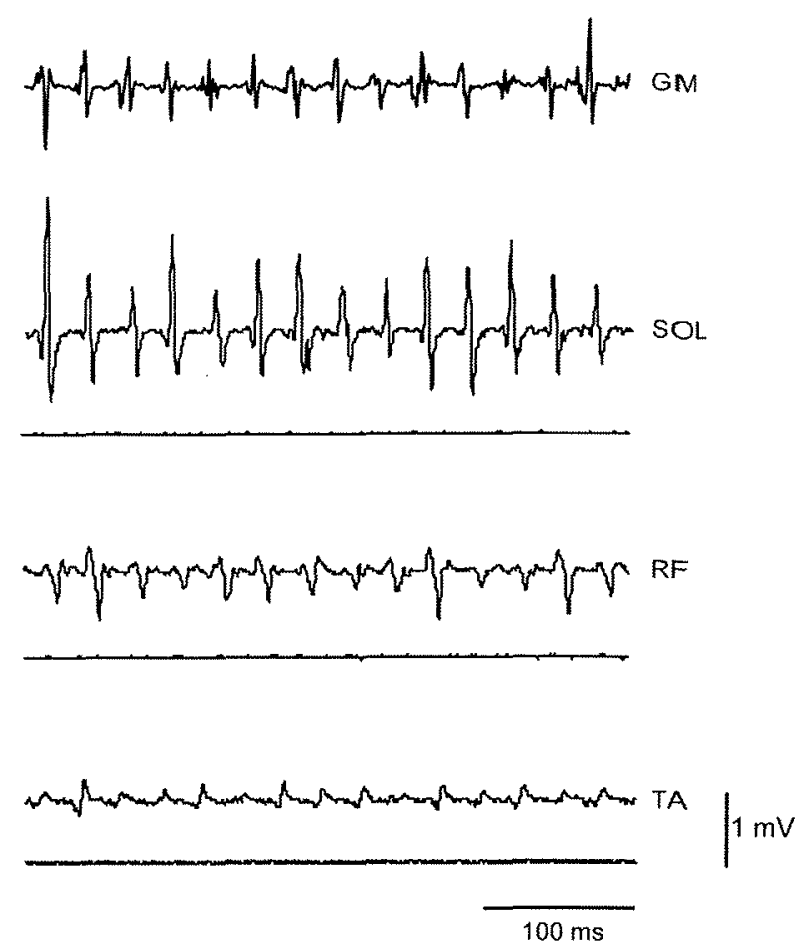

Fig. 2 Representative unprocessed electromyographic activity of GM, SOL, RF, TA (from top to bottom) and the corresponding dummy signals (almost flat line below the respective muscle signalexcept for GM, because no GM dummy was used) during WBV exposure with a vibration frequency of $30 \mathrm{~Hz}$

function (Fig. 3c) are illustrated. The choice of a sawtooth function is arbitrary and only serves to clarify that a signal without any noise and only one obvious frequency component (in this case $25 \mathrm{~Hz}$ ) can have a frequency spectrum with lots of other frequency components, depending on the shape of the signal.

3. Latencies The electromyographic activity during WBV is a periodic one, and the number of peaks per second in the EMG concurs exactly with the preset vibration frequency. The comparison of the latencies of SOL, RF and TA and their respective dummy "latencies" showed that the muscle latencies were independent of the vibration frequency, reflected by their small standard deviations. In contrast, the "latencies" of the dummy electrodes were unsystematic and consequently had large standard deviations (Fig. 4). The averaged latency in SOL was $37 \pm 1 \mathrm{~ms}$ and in SOL dummy $17 \pm 18 \mathrm{~ms}$ (statistically significant difference of the mean as well as the SD, $p<0.001$ ), in RF $27 \pm 2 \mathrm{~ms}$ and in $\mathrm{RF}$ dummy $11 \pm 17 \mathrm{~ms}$ $(p<0.001)$, in TA $34 \pm 2 \mathrm{~ms}$ and in TA dummy $15 \pm 19 \mathrm{~ms}(p<0.001)$. The comparison of the EMG data of SOL and GM in the ankle ergometer and during WBV (Fig. 5) revealed only very small differences 
Fig. 3 a Representative EMG signal of SOL stretch reflex responses evoked in the ankle ergometer and the mean of the corresponding frequency spectrograms of all participants. b EMG signal of SOL during WBV with $25 \mathrm{~Hz}$ and the mean of the corresponding frequency spectra of all participants. $\mathrm{c}$ A sawtooth function with $25 \mathrm{~Hz}$ and its spectrogram. All three spectrograms show peaks at the excitation frequency $(25 \mathrm{~Hz})$ and its harmonics (i.e. 50, 75, $100 \ldots \mathrm{Hz}$ ). d Representative signal of the SOL dummy during WBV with $25 \mathrm{~Hz}$ and the mean of the corresponding frequency spectra of all participants
A

Stretch reflexes
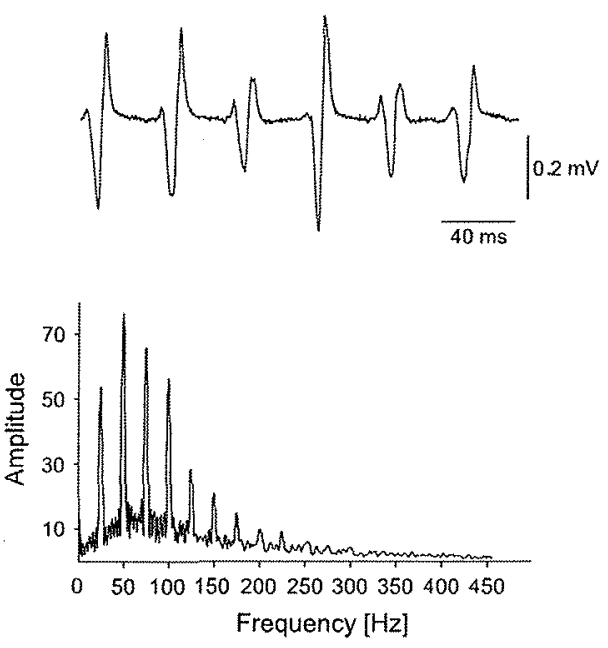

C
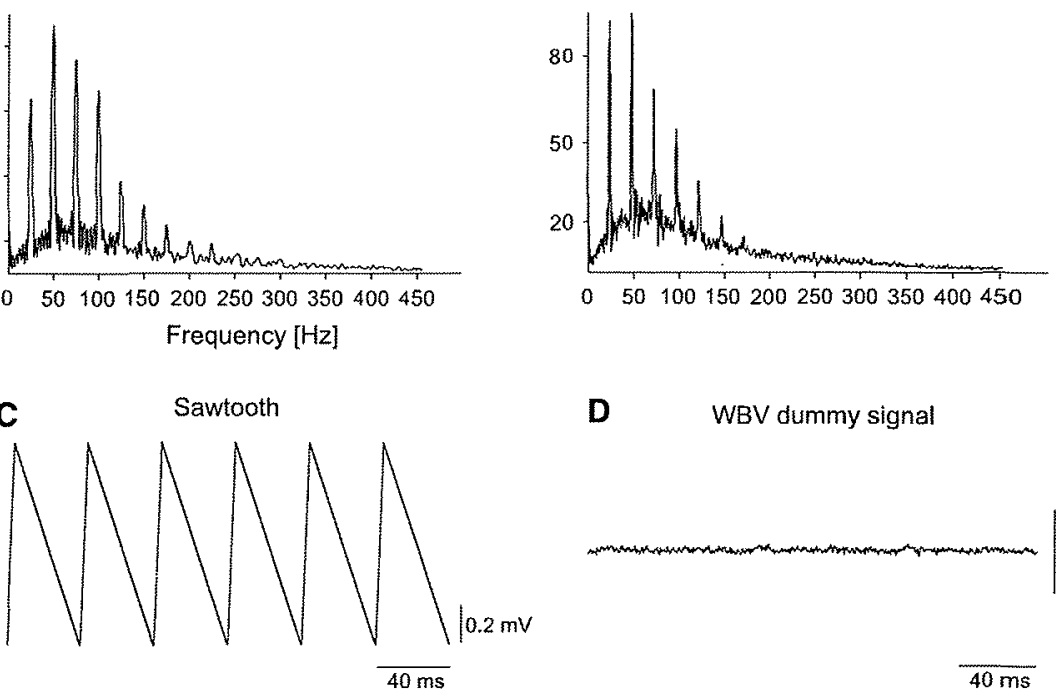

D WBV dummy signal
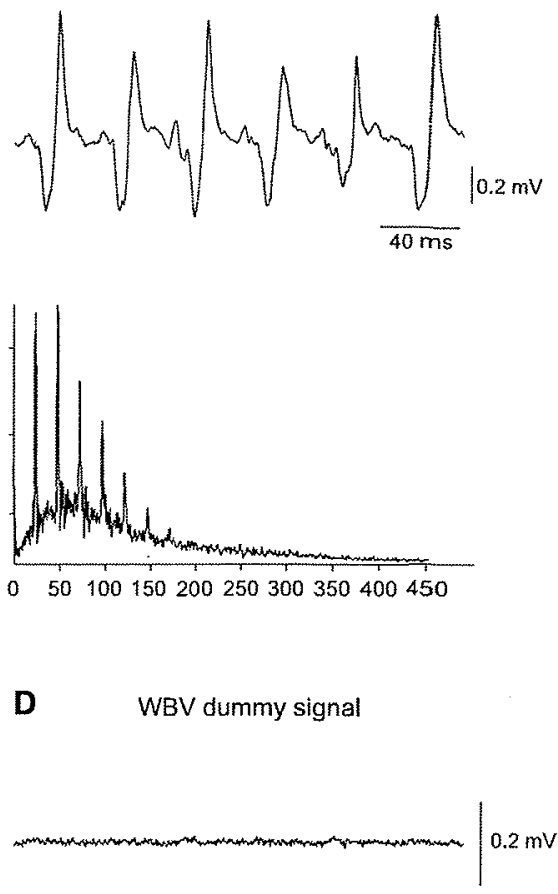
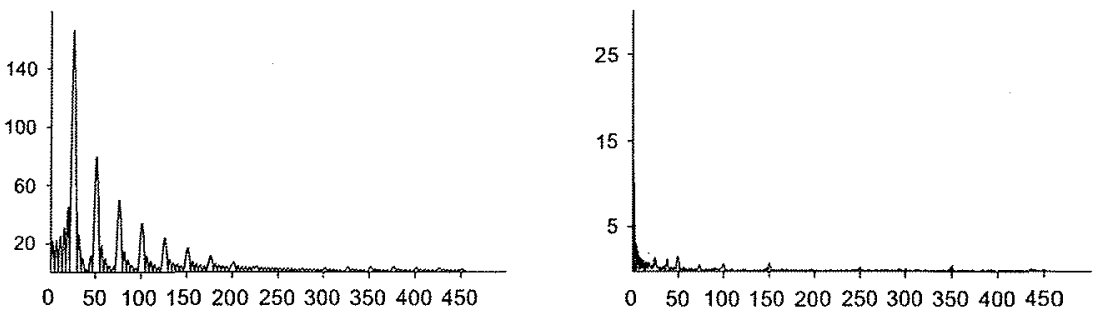

regarding the latencies: eight in ten participants had identical SOL latencies, the other two participants' latencies differed by $<1 \mathrm{~ms}$ (no statistically significant difference of the mean values, $p=0.18$; see Fig. 4). In GM, five participants had identical latencies, the other five participants had a latency difference of $<1 \mathrm{~ms}$ $(p=0.48)$.

4. Cuff experiment The cuff experiment in the ankle ergometer showed a stretch reflex reduction of $56 \pm 13 \%$ of the peak-to-peak value when the cuff was inflated in comparison to the deflated cuff $(p<0.01)$. The same analysis during WBV resulted in a $61 \pm 17 \%$ decline of the peak-to-peak value when the cuff was inflated compared to a deflated cuff $(p<0.001)$.

\section{Discussion}

Experimental data from all four approaches used in this study indicate that WBV most likely elicits stretch reflexes in the leg extensor muscles. Furthermore, the contribution of motion artifacts to the EMG signal during WBV seems to be insignificant.

The first experiment was designed to quantify a potential contribution of motion artifacts to the EMG activity. The results show only a marginal contribution, as indicated by an averaged $\mathrm{D} / \mathrm{M}$ ratio of $7 \%$ in SOL and RF. As demonstrated by the $\mathrm{D} / \mathrm{M}$ ratios of 14 (SOL) and 27 (RF) \% during normal stance, it can be argued that the contribution of motion artifacts is even less because the dummy activity mostly consisted of background noise that was already 


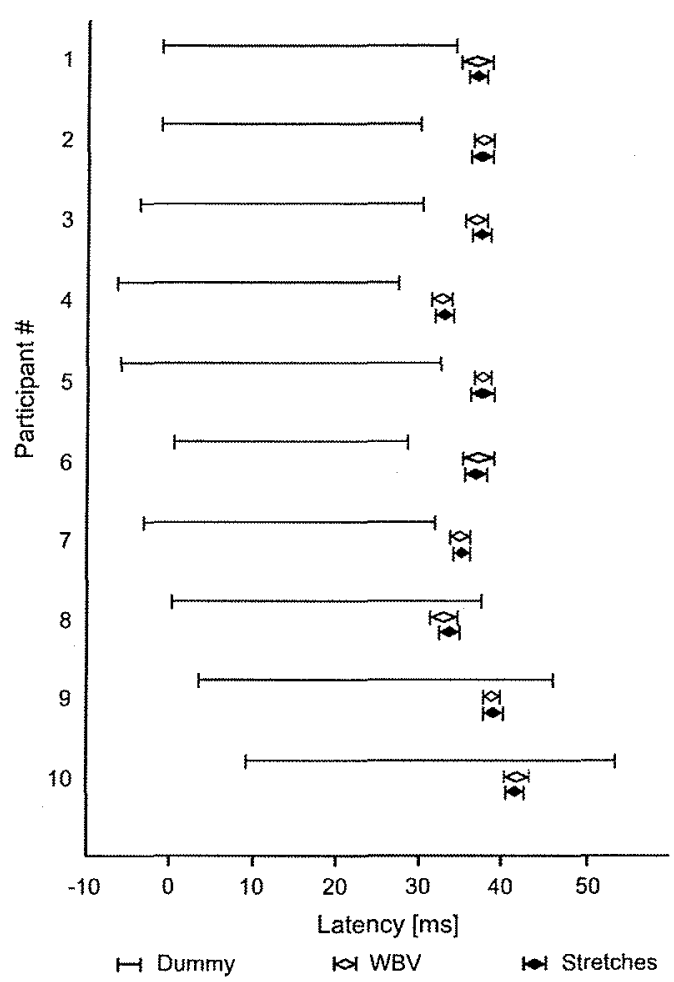

Fig. 4 Averaged SOL stretch reflex latency in the ankle ergometer (mean $\pm \mathrm{SD}$ of 20 stretch reflexes) and latency of the supposed stretch reflex during WBV (mean $\pm \mathrm{SD}$ of all frequencies) for each participant (\#1-10), as well as the "latency" range of the SOL dummy electrodes. The latencies in the ankle ergometer are almost identical to the latencies during WBV and their standard deviations are very small, whereas the latency range (i.e. the standard deviation) of the dummy electrodes is very wide and quite random

present without vibration. In addition, the $D / M$ ratio decreased with increasing vibration frequency because vibration with low frequencies led to a similarly low muscular activity as during normal stance. Thus, it is most likely that the relatively high $\mathrm{D} / \mathrm{M}$ ratios during low frequency vibration were caused by low muscular activity and not by high dummy activity. In contrast to the results obtained by Fratini and co-workers (2009), our "dummy" results indicate only minor contribution of motion artifacts during WBV. This discrepancy can most likely be explained by methodological constraints in the earlier study (Fratini et al. 2009). Fratini and co-workers placed electrodes on the patella, assuming that any signal recorded from those electrodes during WBV had to be an artifact. However, our preliminary tests revealed that a considerable amount of crosstalk is recorded from electrodes placed over the patella or the tibia plateau. For instance, during slow voluntary contractions, which certainly did not cause any significant amount of motion artifacts, the measured tibia signal contained $>50 \%$ of the EMG signal from TA. In our opinion, it is therefore not possible to infer the contribution of motion artifacts from electrodes placed above osseous structures.

The comparison of the dummy "latencies" (in case the dummy signal did not exclusively consist of background noise but had singular peaks) and the latencies of the supposed stretch reflexes showed no similarities at all (Fig. 4). Therefore, a connection between the small unsystematic spikes seen in the dummy signals and the big systematic responses in the muscle signals seems to be unlikely. On the other hand, the latencies of the supposed stretch reflexes during WBV and the latencies of the stretch reflexes evoked in the ankle ergometer were both systematic and almost identical, suggesting a similar mechanism. In addition, the latencies during WBV were determined for all vibration trials and then averaged for each participant. The resulting small standard deviations revealed that the latencies were independent of the vibration frequency, indicating a physiological mechanism. Finally, the measured latencies during WBV corresponded well to previously reported latencies of the mechanically induced stretch reflex responses in the triceps surae (see for example, Gruber et al. 2007). The comparison of the responses elicited during $W B V$ and during trials in the ankle ergometer therefore point towards a strong contribution of stretch reflex activity to the overall muscular activity during WBV.

If this was the case, the EMG signal during WBV should be very similar to the EMG signal of stretch reflexes evoked in the ankle ergometer, which is why we examined the spectrograms of those signals. However, it has to be emphasized that this comparison can only verify a necessary criterion for the congruence of the two signals, not a sufficient one. This is due to the genesis and purpose of a spectrogram: normally it is generated by applying a Fourier transformation to the signal of interest, i.e. by approximating the signal by a sum of sine and cosine functions with different frequencies and coefficients (see mathematical textbooks, for example Stein and Shakarchi 2003). Hence, a frequency spectrum visualizes the contribution of the different sine and cosine functions to the approximated signal. If the examined signal is not a wave signal, this visualization cannot provide much additional information. For example, two similar spectrograms can belong to two completely different signals, mainly because the phase information is lost when using this kind of visualization. However, if two spectrograms are quite different, the original signals have to differ considerably too, i.e. a spectrogram is a tool that can reveal differences (because it is a necessary criterion) but cannot demonstrate the congruence of two signals (because it is not a sufficient criterion). In the present study, the spectrograms of the dummy signals were quite different from the spectrograms of the corresponding EMG signals, whereas the frequency spectra of 
Fig. 5 Averaged EMG activity position of one participant in the ankle ergometer (top) during WBV with $10 \mathrm{~Hz}$ (second), $20 \mathrm{~Hz}$ (third) and $30 \mathrm{~Hz}$ identical latencies (time from the low point of the position signal to the onset of the EMG activity) in all cases (mean $\pm \mathrm{SD}$ ) and platform (bottom). Note the almost

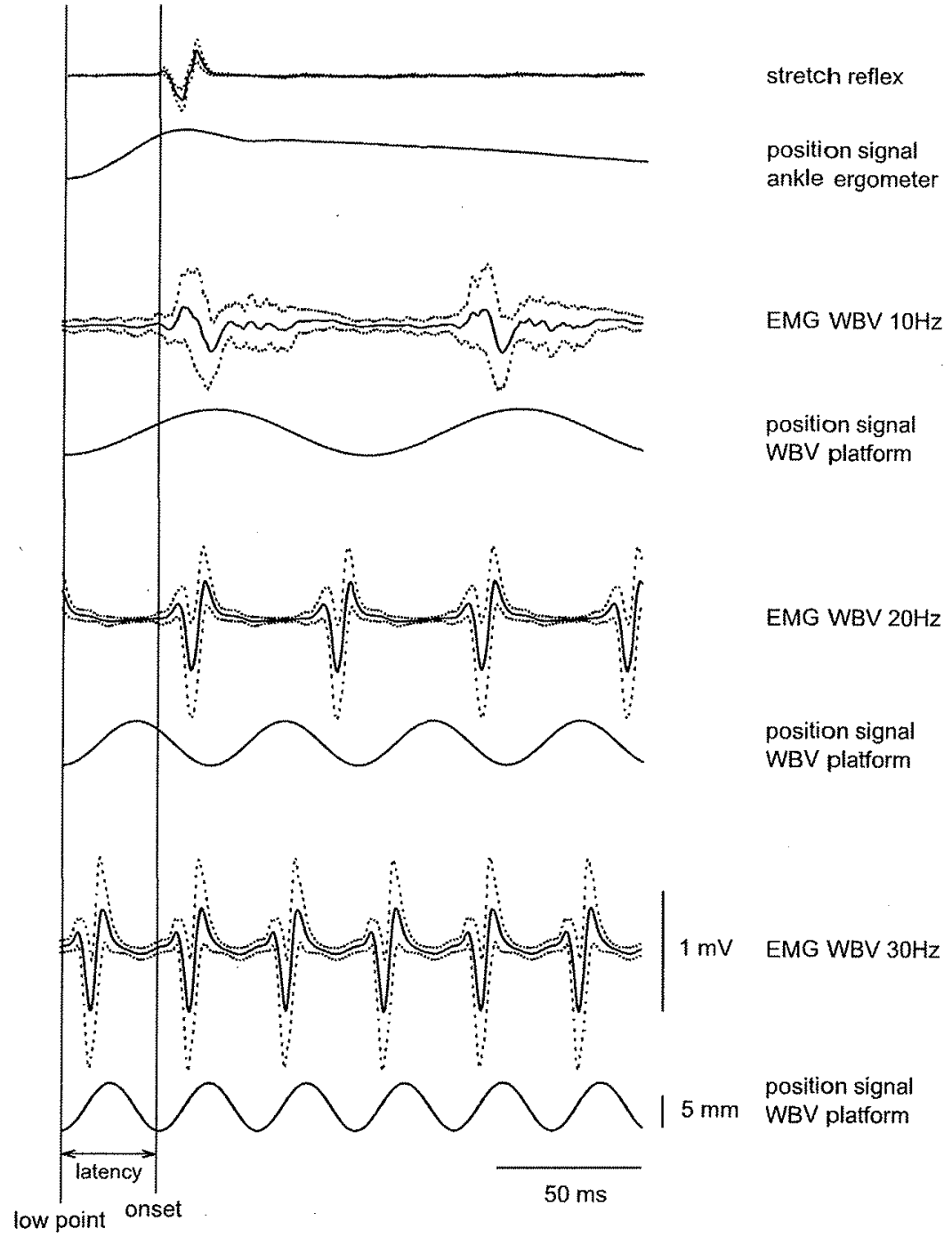

the mechanically evoked stretch reflexes and the EMG activity during WBV are very similar, indicating the possibility that WBV elicits stretch reflexes, just like the direct comparison of the original signals suggests. It also shows that determining filter methods based only on the shape of the spectrogram (Abercromby et al. 2007; Mileva et al. 2009) is not adequate: if one would filter the peaks corresponding to the excitation frequency and its harmonics, the signal would be altered significantly, i.e. a stretch reflex (or sawtooth or any other signal with high peaks in its spectrogram) would not be recognizable afterwards because part of its shape is encoded in the frequency distribution (the other part is encoded in the phase space).

The cuff experiment was based on the study by Leukel and co-workers (2009). They demonstrated that a pressure application provoked by the inflation of a blood pressure cuff can cause a selective and immediate significant reduction of the short-latency response of the stretch reflex. We hypothesized that if the muscular activity during WBV is caused by stretch reflexes, short-term pressure application should be able to reduce the amplitudes of the shortlatency responses and thus the overall EMG activity during WBV. In the present experiment, the peak-to-peak values in the EMG during WBV could be reduced by $>50 \%$ by inflating the cuff, which is another indication that the EMG activity during WBV consists at least partly of stretch reflexes, as motion artifacts arising from shaking cables would not have been affected by the inflation of the cuff.

Although all the results point towards WBV-induced stretch reflexes and only minor influence of motion artifacts, one limitation of this study might be that the experiments are not very suitable to detect motion artifacts arising from a possible movement of electrodes relatively to the skin. However, this is a general problem associated 
with any EMG recording of dynamic movements, e.g. jumps or running. Furthermore, one could argue that the results of the dummy experiment could be influenced by the type of equipment used, i.e. if preamplified or reusable electrodes produce the same results as the non-preamplified disposable ones used in this study. This is not very likely, because preamplified electrodes are designed to be even less susceptible to noise or artifacts than non-preamplified electrodes, but nevertheless it is a possibility that cannot be excluded by this study. Fortunately, the study's strongest evidence stems from the latency comparisons which are not equipment dependent.

\section{Conclusion}

The results of our experiments show that the main part of the periodic electromyographic activity of the examined leg extensor muscles (soleus, gastrocnemius medialis and rectus femoris muscles) seems to be caused by vibrationinduced stretch reflexes. Furthermore, it is possible to use EMG data recorded during WBV without applying additional filters because the contribution of motion artifacts seems to be insignificant. The finding that WBV seems to elicit stretch reflexes may have implications for the use of WBV as a form of training for sports or specifically designed rehabilitation programs. However, future studies have to clarify whether the huge number of stretches and stretch reflexes induced during WBV has any functional relevance.

\section{References}

Abercromby AF, Amonette WE, Layne CS, McFarlin BK, Hinman MR, Paloski WH (2007) Variation in neuromuscular responses during acute whole-body vibration exercise. Med Sci Sports Exerc 39:1642-1650

Blottner D, Salanova M, Puttmann B, Schiffl G, Felsenberg D, Buehring B, Rittweger J (2006) Human skeletal muscle structure and function preserved by vibration muscle exercise following 55 days of bed rest. Eur J Appl Physiol 97:261-271

Burke D, Hagbarth KE, Lofstedt L, Wallin BG (1976) The responses of human muscle spindle endings to vibration of non-contracting muscles. J Physiol 261:673-693
Cochrane DJ, Stannard SR (2005) Acute whole body vibration training increases vertical jump and flexibility performance in elite female field hockey players. Br J Sports Med 39:860-865

De Ruiter CJ, Van Raak SM, Schilperoort JV, Hollander AP, de Haan $A(2003 a)$ The effects of 11 weeks whole body vibration training on jump height, contractile properties and activation of human knee extensors. Eur J Appl Physiol 90:595-600

De Ruiter CJ, van der Linden RM, van der Zijden MJ, Hollander AP, de Haan A (2003b) Short-term effects of whole-body vibration on maximal voluntary isometric knee extensor force and rate of force rise. Eur J Appl Physiol 88:472-475

Fratini A, Cesarelli M, Bifulco P, Romano M (2009) Relevance of motion artifact in electromyography recordings during vibration treatment. J Electromyogr Kinesiol 19:710-718

Gollhofer A, Rapp W (1993) Recovery of stretch reflex responses following mechanical stimulation. Eur J Appl Physiol Occup Physiol 66:415-420

Gruber M, Taube W, Gollhofer A, Beck S, Amtage F, Schubert M (2007) Training-specific adaptations of $\mathrm{H}$ - and stretch reflexes in human soleus muscle. J Mot Behav 39:68-78

Kvorning T, Bagger M, Caserotti P, Madsen K (2006) Effects of vibration and resistance training on neuromuscular and hormonal measures. Eur J Appl Physiol 96:615-625

Leukel C, Lundbye-Jensen J, Zuur AT, Gruber M, Gollhofer A, Taube W (2009) Short-term pressure induced suppression of the short latency response-a new methodology for investigating stretch reflexes. J Appl Physiol 107:1051-1058

Matthews PB (1966) The reflex excitation of the soleus muscle of the decerebrate cat caused by vibration applied to its tendon. J Physiol 184:450—472

Mester J, Spitzenpfeil P, Yue Z (2002) Vibration loads: potential for strength and power development. In: Komi $P$ (ed) Strength and power in sport, 2nd edn. Blackwell, Oxford, pp 488-501

Mileva KN, Bowtell JL, Kossev AR (2009) Effects of low-frequency whole-body vibration on motor-evoked potentials in healthy men. Exp Physiol 94:103-116

Rittweger J, Beller G, Felsenberg D (2000) Acute physiological effects of exhaustive whole-body vibration exercise in man. Clin Physiol 20:134-142

Rittweger J, Mutschelknauss M, Felsenberg D (2003) Acute changes in neuromuscular excitability after exhaustive whole body vibration exercise as compared to exhaustion by squatting exercise. Clin Physiol Funct Imaging 23:81-86

Roll JP, Vedel JP, Ribot E (1989) Alteration of proprioceptive messages induced by tendon vibration in man: a microneurographic study. Exp Brain Res 76:213-222

Stein E, Shakarchi R (2003) Fourier analysis: an introduction. Princeton University Press, Princeton

Torvinen S, Sievanen H, Jarvinen TA, Pasanen M, Kontulainen S, Kannus P (2002) Effect of 4-min vertical whole body vibration on muscle performance and body balance: a randomized crossover study. Int J Sports Med 23:374-379 